\title{
A Novel Three Dimension Autonomous Chaotic System with a Quadratic Exponential Nonlinear Term
}

\author{
Fei $\mathrm{Yu}$ \\ College of Information Science and Engineering \\ Hunan University \\ Changsha, China \\ yufeiyfyf@yahoo.com.cn
}

\author{
Chunhua Wang \\ College of Information Science and Engineering \\ Hunan University \\ Changsha, China \\ wch1227164@sina.com
}

\begin{abstract}
A novel three dimension autonomous (3D) chaotic system with a quadratic exponential nonlinear term and a quadratic cross-product term is described in this paper. The basic dynamical properties of the new attractor are studied. The forming mechanism of its compound structure, obtained by merging together two simple attractors after performing one mirror operation, has been investigated by detailed numerical as well as theoretical analysis. Finally, the exponential operation circuit and its temperature-compensation circuit, which makes the new system more applicable from a practical engineering perspective, are investigated.
\end{abstract}

Keywords- 3D chaotic system; exponential nonlinear term; exponential operation circuit; temperature-compensation circuit

\section{INTRODUCTION}

Since Lorenz found the first chaotic attractor in a three firstorder autonomous ordinary differential equations (ODEs) when he studied the atmospheric convection in 1963 [1], many new three dimension (3D) chaotic attractors have been proposed in the last three decades, such as the Rossler system [2], the Chen system [3], the Lü system [4], the Liu system [5], and the generalized Lorenz system family [6]. The complicated dynamic properties of these chaotic systems are all obtained by some quadratic cross-product nonlinearity terms at the righthand side in ODEs.

Recently, Wei and Yang [7] revealed a 3D autonomous chaotic attractor with a nonlinear term in the form of exponential function at the right-hand side in ODEs as $\dot{x}=a y-a x, \dot{y}=-b y+m x z, \dot{z}=n-e^{x y}$, where the existence of singularly degenerate heteroclinic cycles for a suitable choice of the parameters was investigated. Chaotic attractors with a quadratic exponential nonlinear term in three ODEs have never been found so far.

In this paper, a novel chaotic attractor is proposed. It is a $3 \mathrm{D}$ autonomous system which relies on a quadratic exponential nonlinear term and a quadratic cross-product term to introduce the nonlinearity necessary for folding trajectories. The chaotic attractor obtained from the new system according to the detailed numerical as well as theoretical analysis is also the two-scroll attractor, exhibiting complex chaotic dynamics [5].
The chaotic attractor is similar to Lorenz chaotic attractor, but not equivalent in the topological structure. Nonlinear dynamic properties of this system are studied by means of nonlinear dynamics theory, numerical simulation, Lyapunov exponents, Poincare mapping, fractal dimension, continuous spectrum and bifurcation diagram. The compound structure of the two-scroll attractor obtained by merging together two simple attractors after performing one mirror operation is explored here. Finally, the exponential operation circuit with temperaturecompensation of the new chaotic system is simply designed.

\section{NOVEL 3D AUTONOMOUS CHAOTIC SYSTEM}

A novel 3D autonomous chaotic system is expressed as follows:

$$
\left\{\begin{array}{l}
\dot{x}=a(y-x), \\
\dot{y}=b x-c x z, \\
\dot{z}=e^{x y}-d z,
\end{array}\right.
$$

where $a, b, c, d$ are all constants coefficients assuming that $a, b, c, d>0$ and $x, y, z$ are the state variables. There are six terms on the right-hand side but it mainly relies on two quadratic nonlinearities, one is a quadratic exponential nonlinear term and the other is a quadratic cross-product term, namely, $e^{x y}$ and $x z$, respectively.

System (1) can generate a new chaotic attractor for the parameters $a=10, b=40, c=2, d=2.5$ with the initial conditions $[2.2,2.4,28]^{T}$. The chaotic attractor is displayed in Figure 1. It appears that the new attractor exhibits the interesting complex and abundant of the chaotic dynamics behavior, which is similar to Lorenz chaotic attractor, but is different from that of the Lorenz system or any existing systems. 


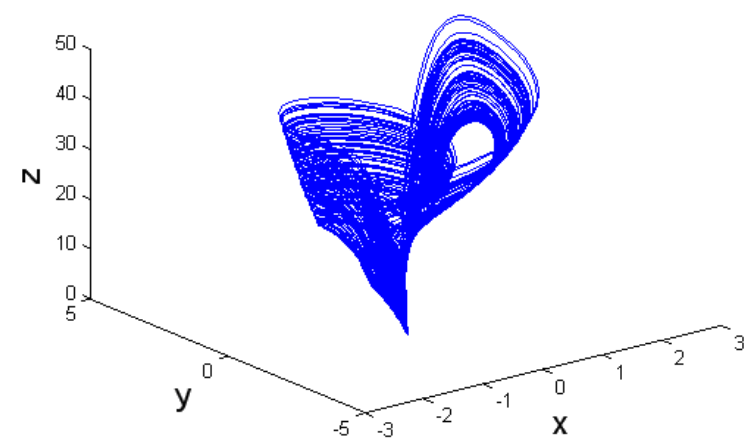

Fig. 1. Phase portraits of the new attractor.

\section{BASIC PROPERTIES OF THE NEW SYSTEM}

\section{A. Equilibria}

Let:

$$
\left\{\begin{array}{l}
a(y-x)=0 \\
b x-c x z=0 \\
e^{x y}-d z=0
\end{array}\right.
$$

If $d b>c$ and $c \neq 0$, the system has two equilibria points, which are respectively described as follows:

$$
\begin{gathered}
E^{+}(\sqrt{\ln (d b / c)}, \sqrt{\ln (d b / c)}, b / c), \\
E^{-}(-\sqrt{\ln (d b / c)},-\sqrt{\ln (d b / c)}, b / c) .
\end{gathered}
$$

When $a=10, b=40, c=2, d=2.5$, we operate above those nonlinear algebraic equations and obtain that:

$$
E^{+}(1.978,1.978,20), E^{-}(-1.978,-1.978,20)
$$

For equilibrium point $E^{+}$, system (1) are linearized, the Jacobian matrix is defined as:

$$
J^{+}=\left(\begin{array}{ccc}
-a & a & 0 \\
b-c z & 0 & -c x \\
y e^{x y} & x e^{x y} & -d
\end{array}\right)=\left(\begin{array}{ccc}
-10 & 10 & 0 \\
0 & 0 & -3.956 \\
30.278 & 30.278 & -2.5
\end{array}\right) \text {. }
$$

To gain its eigenvalues, we let $\left|\lambda I-J^{+}\right|=0$. These eigenvalues corresponding to the equilibrium point $E^{+}$are $\lambda_{1}=-14.192, \lambda_{2}=0.846+12.965 i$ and $\lambda_{3}=0.846-12.965 i$.

Here $\lambda_{1}$ is a negative real number, $\lambda_{2}$ and $\lambda_{3}$ become a pair of complex conjugate eigenvalues with positive real parts.
The equilibrium point $E^{+}$is a saddle-focus point. And system (1) is unstable at this equilibrium point.

And then, for the equilibrium point $E^{-}$, its Jacobian matrix equals to:

$$
J^{-}=\left(\begin{array}{ccc}
-a & a & 0 \\
b-c z & 0 & -c x \\
y e^{x y} & x e^{x y} & -d
\end{array}\right)=\left(\begin{array}{ccc}
-10 & 10 & 0 \\
0 & 0 & 3.956 \\
-30.278 & -30.278 & -2.5
\end{array}\right) .
$$

The same we let $\left|\lambda I-J^{-}\right|=0$. These eigenvalues corresponding to the equilibrium point $E^{-}$are $\lambda_{1}=-14.192$, $\lambda_{2}=0.846+12.965 i$ and $\lambda_{3}=0.846-12.965 i$.

Apparently $\lambda_{1}$ is a negative real number, $\lambda_{2}$ and $\lambda_{3}$ form a complex conjugate pair and their real parts are positive. The equilibrium point $E^{-}$is also a saddle-focus point. And system (1) is unstable at this equilibrium point.

By the above brief analysis, the two equilibrium points of the non-linear system are all saddle focus-nodes.

\section{B. Symmetry and invariance}

It is easy to see the invariance of system under the coordinate transformation $(x, y, z) \rightarrow(-x,-y, z)$, i.e., the system has rotation symmetry around the $z$-axis [7].

\section{Dissipativity}

The three Lyapunov exponents and the divergence of the vector field is:

$$
\sum_{i=1}^{3} L E_{i}=\Delta V=\frac{\partial \dot{x}}{\partial x}+\frac{\partial \dot{y}}{\partial y}+\frac{\partial \dot{z}}{\partial z}=-(a+d)=f
$$

where $L E_{i}(i=1,2,3)$ denote the three Lyapunov exponents of the system. Note that $f=-(a+d)=-12.5$ is a negative value, so the system is a dissipative system and an exponential rate is:

$$
\frac{d V}{d t}=e^{f}=e^{-12.5}
$$

From (4), it can be seen that a volume element $V_{0}$ is contracted by the flow into a volume element $V_{0} e^{-12.5 t}$ in time $t$. This means that each volume containing the system trajectory shrinks to zero as $t \rightarrow \infty$ at an exponential rate of -12.5 . Therefore, all system orbits are ultimately confined to a specific subset having zero volume and the asymptotic motion settles onto an attractor [8]. 


\section{Lyapunov exponent and fractional dimension}

The Lyapunov exponents generally refer to the average exponential rates of divergence or convergence of nearby trajectories in the phase space. If there is at least one positive Lyapunov exponent, the system can be defined to be chaotic. According to the detailed numerical as well as theoretical analysis and (3), the Lyapunov exponents are found to be $l_{1}=1.459, l_{1}=0$ and $l_{3}=-13.959$. Therefore, the Lyapunov dimension of this system is:

$$
D_{L}=j+\frac{\sum_{i=1}^{j} l_{i}}{\left|l_{j+1}\right|}=2+\frac{l_{1}+l_{2}}{\left|l_{3}\right|}=2+\frac{1.459}{|-13.959|}=2.104
$$

Equation (5) means system (1) is really a dissipative system, and the Lyapunov dimensions of the system are fractional. Having a strange attractor and positive Lyapunov exponent, it is obvious that the system is really a $3 \mathrm{D}$ chaotic system.

\section{E. Spectrum map, time domain and Poincare maps}

The spectrum of system (1) exhibits a continuous broadband feature as shown in Figure 2. Figure 3 shows that the evolution of the chaos trajectories is very sensitive to initial conditions [9]. The initial values of the system are set to $[2.2,2.399,28]^{T}$ for the solid line and $[2.2,2.4,28]^{T}$ for the dashed line.

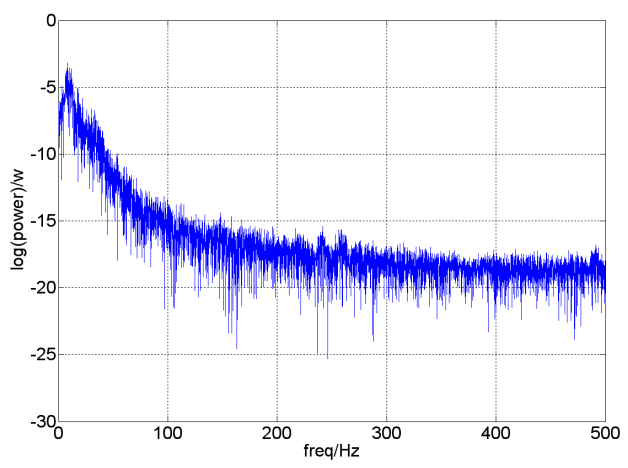

Fig. 2. Spectrum of $\log |x|$

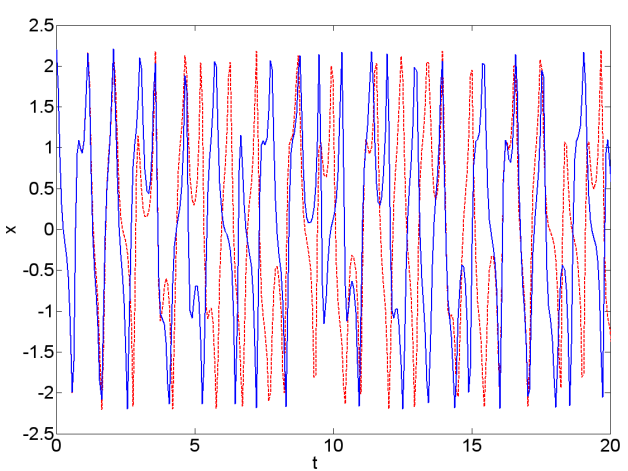

Fig. 3. Sensitivity of system (1)
The Poincare maps are shown in Figure 4. From Figure 4(a)-(b), it can be seen that the Poincare maps consist of virtually symmetrical branches and a number of nearly symmetrical twigs. It is also found that the section of the attractor looks like some circles from Poincare map as Figure 4(c)-(d).

\section{F. The influence of system parameters}

From the above analysis, it is visible that the stability of system equilibria will be changed along with the change of system parameters, and the system will also be in different state. By using numerical simulation method, the change of system parameters and system conditions are analyzed below. We let $a$ increasing when other parameters are fixed.

Figures 5 and 6 show the bifurcation diagram and Lyapunov exponent spectrum versus increasing $a$. While $a$ increases, the system is undergoing some representative dynamical routes, such as stable fixed points, chaos, quasi-periodic loops and period-doubling bifurcation, which are summarized as follows:

- $0<a \leq 1.35$, system (1) is stable, as shown in Figure $7(\mathrm{a})$.

- $1.35<a \leq 12$, system (1) is chaotic, and there are several periodic windows in the chaotic band, as shown in Figure 7(b)-(c).

- $\quad 12<a \leq 22$, systems (1) are some quasi-periodic loops and also call a reverse period-doubling bifurcation window, as shown in Figure 7(d).

- $22<a \leq 28.9$, system (1) is chaotic, and there are several periodic windows in the chaotic band, as shown in Figure 7(e).

- $28.9<a \leq 40$, there is a reverse period-doubling bifurcation window too, as shown in Figure 7(f).

\section{G. Forming mechanism of this new chaotic attractor structure}

Compound structures of the new system (1) may be obtained by merging together two simple attractors after performing one mirror operation. Such an operation can be revealed through the use of a controlled system of the form:

$$
\left\{\begin{array}{l}
\dot{x}=a(y-x) \\
\dot{y}=b x-c x z+k y+u \\
\dot{z}=e^{x y}-d z
\end{array}\right.
$$

where $u$ is a parameter of control and the value of $u$ can be changed within a certain range. Here, we still select the initial values of the system as $[2.2,2.4,28]^{T}$. When $u=3$, the attractor evolves into partial but is still bounded in this time, the corresponding strange attractors are shown in Figure 8a. When $u=5$, the attractors are evolved into the single right scroll attractor, it is only one half the original chaotic attractors 
in this time, the corresponding strange attractors are shown in Figure $8 b$.

Then we select $u$ to be a negative value. When $u=-3$, the corresponding strange attractors are shown in Figure $8 \mathrm{c}$, the

a

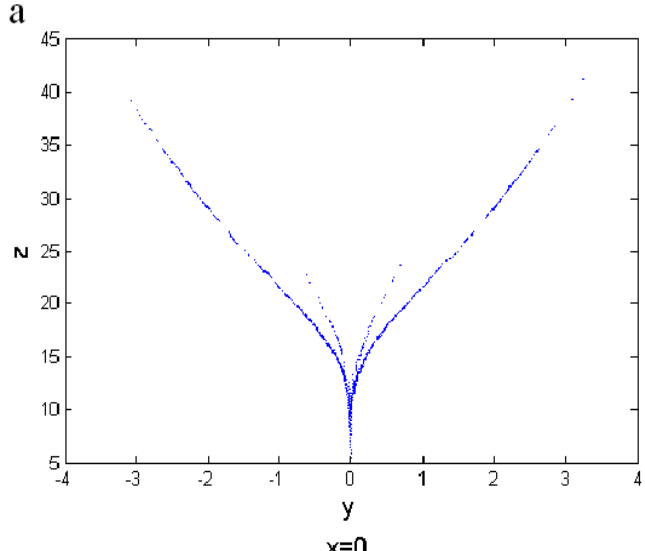

b

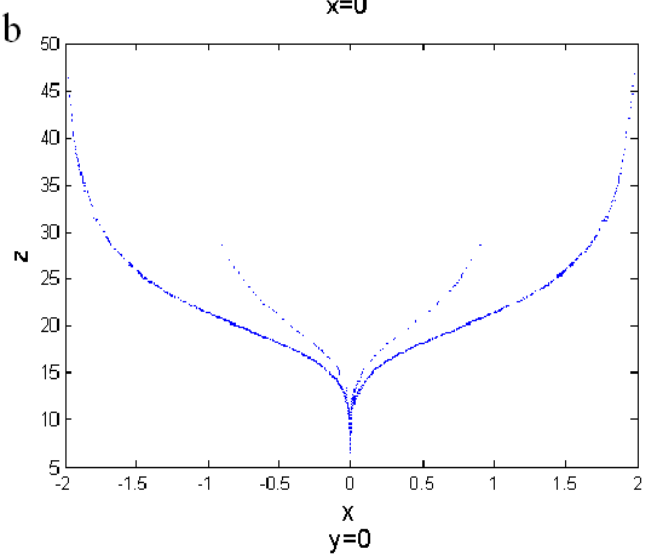

attractor evolves into partial but is still bounded in this time. When $u=-5$, the corresponding strange attractors are shown in Figure 8d, the attractors are evolved into the single left scroll attractor; it is only one half the original chaotic attractors in this time.

$\mathrm{c}$

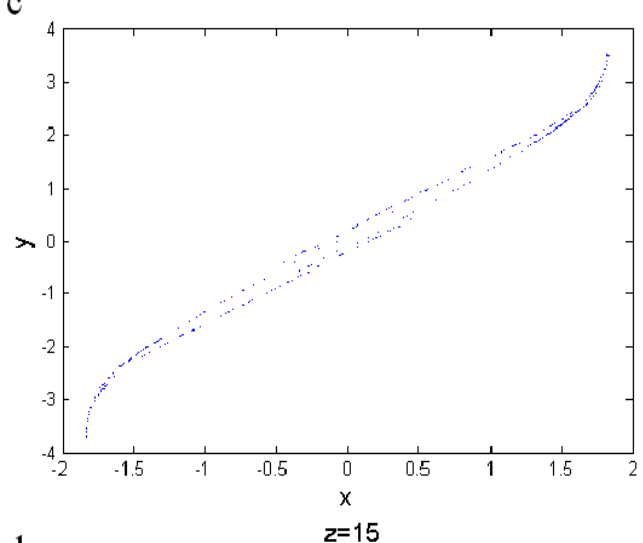

d

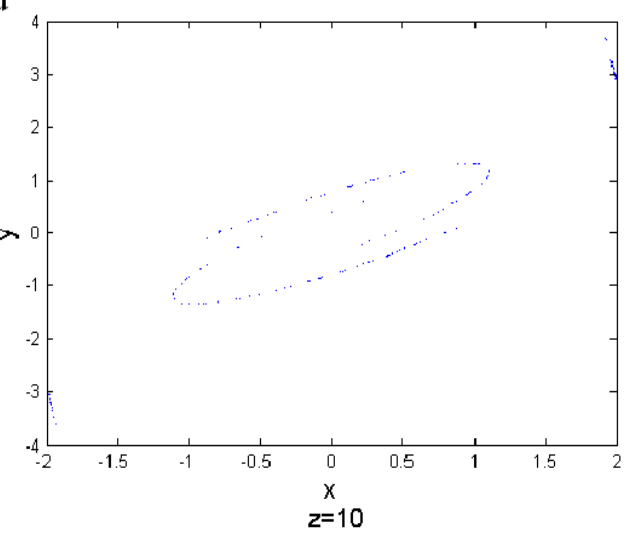

Fig. 4. Poincaré maps in planes where (a) $x=0$, (b) $y=0$, (c) $z=15$, (d) $z=10$

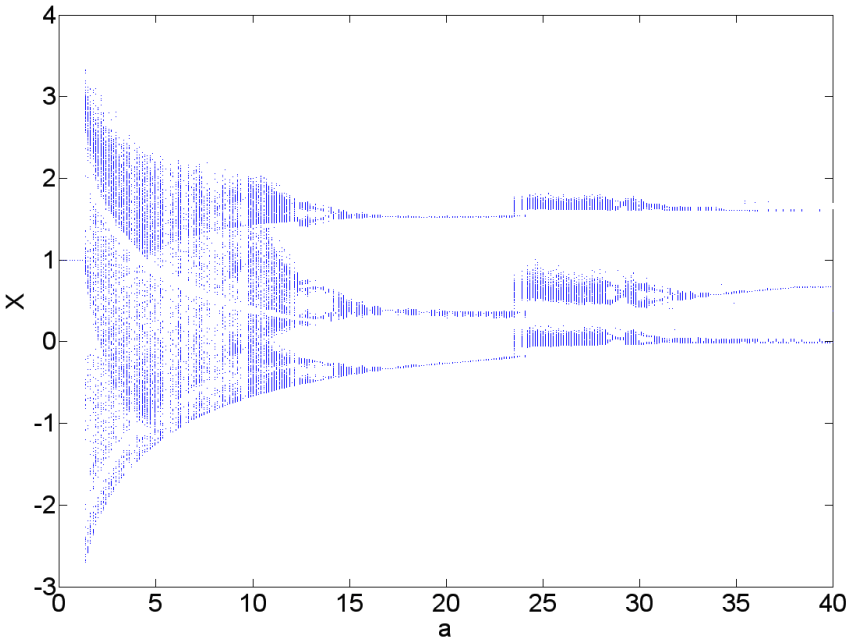

Fig. 5. Bifurcation diagram of system (1) with $0<\mathrm{a}<40$

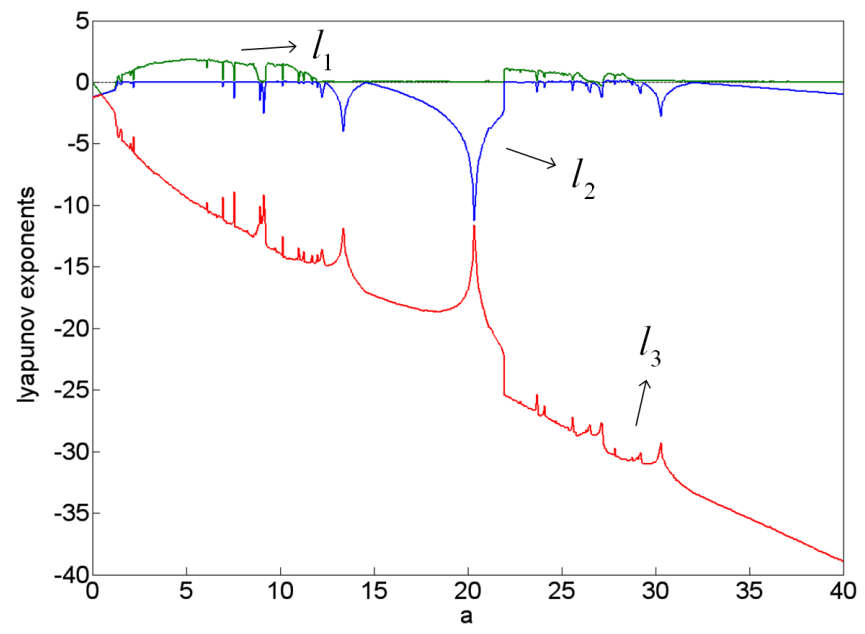

Fig. 6. Lyapunov exponents spectrum of system (1) with $0<a<40$ 


\section{EXPONENTIAL OPERATION CIRCUIT}

The exponential operation circuit is simply studied in this part. First, using the semiconductor PN-junction exponential
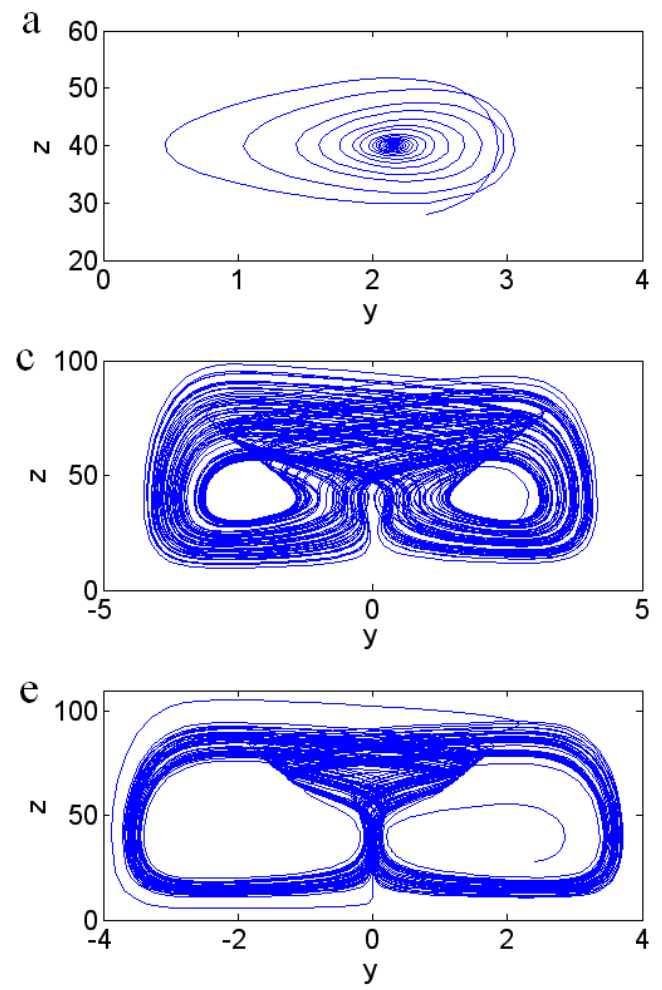

volt-ampere characteristic, the exponential operation can be realized. Then, in order to suppress temperature-drift, the technology of temperature-compensation is used in the exponential operation circuit.
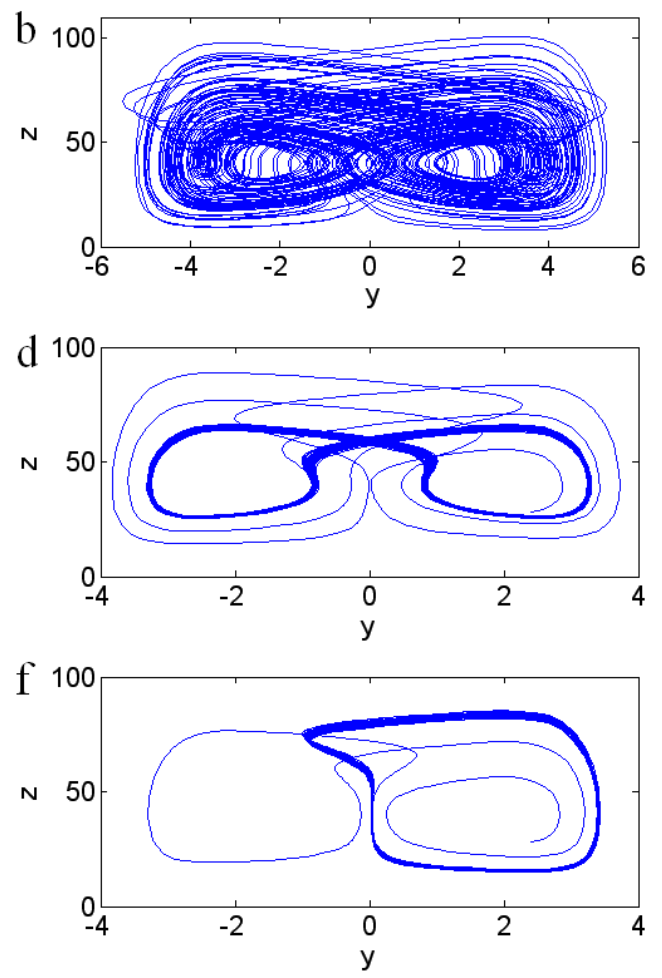

Fig. 7. Phase portraits of system (1) in $y-z$ plane with $(b, c, d)=(40,2,2.5)$ at initial values $[2.2,2.4,28]^{T}:$ (a) $a=1$, (b) $a=3$, (c) $a=10$, (d) $a=15$, (e) $a=24$, (f) $a=35$
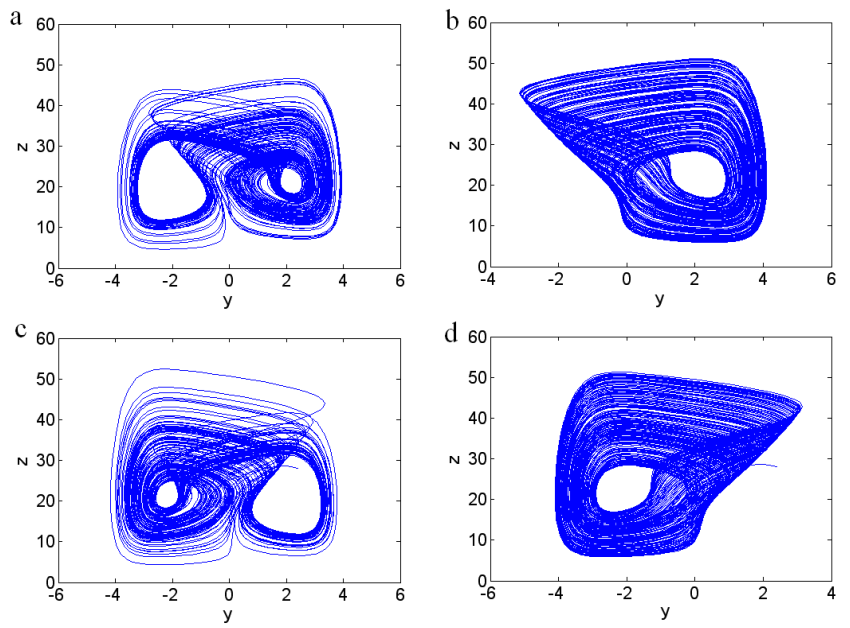

Fig. 8. Phase portraits of system (6) in $y-z$ plane at (a) $u=3$, (b) $u=5$, (c) $u=-3$, (d) $u=-5$.

\section{A. Basic exponential operation circuit}

In actual application, we usually connect the collector with the base of bipolar junction transistor (BJT) to form a diode type. The exponential operation circuit is shown in Figure 9. According to the relationship of $i_{c}$ and $U_{b e}$, we get:

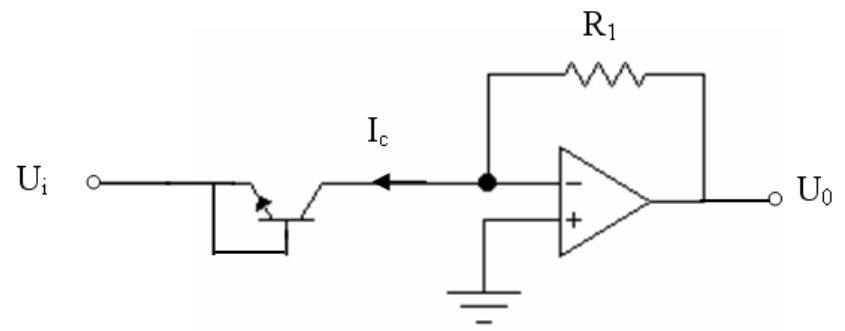

Fig. 9. Exponential operation circuit. 


$$
I_{c}=\alpha I_{e}=\alpha I_{E S}\left(e^{\frac{U_{b e}}{U_{T}}}-1\right)
$$

where $\alpha$ is the current amplification and $\alpha \approx 1, I_{E S}$ and $U_{b e}$ are the transistor parameters. When $U_{b e} \gg U_{T}$, equation (7) is simplified to:

$$
I_{c} \approx I_{E S} e^{\frac{U_{b e}}{U_{T}}}
$$

then

$$
U_{0}=I_{c} R_{1}=I_{E S} R_{1} e^{\frac{U_{b e}}{U_{T}}}=I_{E S} R_{1} e^{\frac{-U_{i}}{U_{T}}} .
$$

\section{B. Exponential operation circuit with temperature- compensation}

As is well known, $I_{E S}$ and $U_{T}$ are functions of the temperature and very sensitive to temperature, so how to improve the temperature stability of the exponential operation circuit is an important problem that needs to be solved in actual application. Here, two methods are applied. First we adopt a pair of BJTs to eliminate the effect of $I_{E S}$, then we use the thermistor to compensate the influence of $U_{T}$. Figure 10 illustrates the exponential operation circuit with temperaturecompensation.

The voltage of point A can be expressed as:

$$
\begin{aligned}
U_{A} & =U_{b e 1}-U_{b e 2} \\
& =U_{T}\left(\ln \frac{U_{r e f}}{I_{E S 1} R_{1}}-\ln \frac{U_{0}}{I_{E S 2} R_{5}}\right) \\
& =U_{T} \ln \frac{U_{r e f} R_{5}}{U_{0} R_{1}},
\end{aligned}
$$

here, the parameters of two BJTs are equal $\left(I_{E S 1}=I_{E S 2}\right)$. $U_{\text {ref }}$ is a reference voltage. When the value of $R_{3}$ was small, the output voltage $U_{0}$ can be expressed as:

$$
U_{0}=\frac{U_{r e f} R_{5}}{R_{1}} e^{-\frac{R_{3}}{R_{3}+R_{4}} \cdot \frac{U_{i}}{U_{T}}},
$$

where $R_{3}$ is the positive temperature coefficient thermistor which use to compensate the temperature variations of $U_{T}$.

\section{CONCLUSION}

This paper presented a novel 3D autonomous chaotic system with a quadratic exponential nonlinear term. Some basic properties of the system have been investigated. In addition, forming mechanisms of compound structures of the new chaotic attractor have been studied and explored. Finally, the exponential operation circuit and its temperaturecompensation circuit of the chaotic system were simply designed.

Despite the abundant and complex dynamical behaviors of the new 3D autonomous system has been discussed in detail in this paper. But even more important, some analysis like control, synchronization and secure communication of the system will be taken into consideration in a future work. Therefore, further research into the system is still important and insightful.

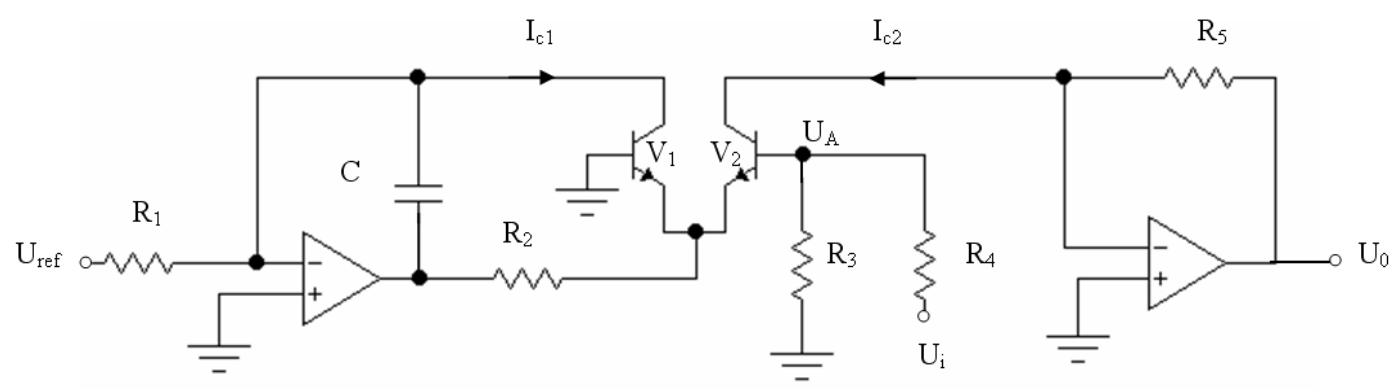

Fig. 10. Exponential operation circuit with temperature-compensation

\section{REFERENCES}

[1] E. N. Lorenz, "Deterministic non-periodic flow", J. Atmos. Sci., Vol. 20, No. 1, pp. 130-141, 1963
[2] O. E . Rossler, "An equation for continuous chaos", Phys. Lett. A, Vol. 57, No. 5, pp. 397-399, 1976

[3] G. Chen, T. Ueta, "Yet another chaotic attractor", Internat. J. Bifur. Chaos, Vol. 9, No. 7, pp. 1465-1457, 1999 
[4] J. Lü, G. Chen, "A new chaotic attractor conined", Internat. J. Bifur. Chaos, Vol. 12, No. 3, pp. 659-662, 2002

[5] C. Liu, T. Liu, L. Liu, K. Liu, "A new chaotic attractor", Chaos, Solitons Fractals, Vol. 22, No. 5, pp. 1031-1038, 2004

[6] S. Celikovsky, G. Chen, "On the generalized Lorenz canonical form", Chaos, Solitons Fractals, Vol. 26, No. 5, pp. 1271-1276, 2005

[7] Z. Wei, Q. Yang, "Dynamical analysis of a new autonomous 3-D chaotic system only with stable equilibria", Nonlinear Anal.: RWA, Vol. 12, No. 1, pp. 106-118, 2011

[8] W. Zhou, Y. Xu, H. Lu, L. Pan, "On dynamics analysis of a new chaotic attractor”, Phys. Lett. A, Vol. 372, No. 36, pp. 5773-5777, 2008

[9] D. Sara, R. M. Hamid, "A novel three-dimensional autonomous chaotic system generating two, three and four-scroll attractors," Phys. Lett. A, Vol. 373, No. 40, pp. 3637-3642, 2009 